\title{
Mise en place du système racinaire du maïs. I. Importance de quelques paramètres relatifs à la plante
}

Marie-Odile JORDAN

I.N.R.A., Station d'Agronomie, Centre de Recherches de Colmar, B.P. 507, F 68021 Colmar

RÉSUMÉ

Ce travail a pour objet de décrire les relations entre organes au cours de la mise en place simultanée des parties aériennes et racinaires chez les graminées et plus particulièrement chez le maïs.

Il a été montré que le nombre de racines primaires dépendait à la fois du patrimoine génétique et de la vigueur des parties aériennes, que les rythmes d'émission racinaires et foliaires sont étroitement corrélés et enfin que le nombre de ramifications secondaires est fonction du diamètre de la racine primaire.

Les paramètres caractéristiques de la croissance : vitesse et taille des organes varient très fortement en fonction des conditions de milieu.

Mots clés additionnels : Relation entre organes, feuille, croissance, facteurs du milieu, parties aériennes.

Growth and development of the corn root system. I. Importance of some parameters concerning the plant.

This work deals with the influence of plant tactors (genotype, growth and development conditions of the roots and of the shoot) on the growth and development of the corn root system. The number of primary roots was found to depend on the genotype and on the vigour of the plants. The emission rhythr of these roots was bound with the phyllochrone, and the number of secondary roots with the diameter of primary roots. Parameters describing root system growth (number and size of organs) depended strongly on surrounding conditions.

Additional key words : Relation between organs, leaf, growth, environmental factor, shoot.

\section{INTRODUCTION}

Si pour les plantes cultivées, la croissance et le développement (notions définies par J. RICARD, 1960) des parties aériennes ont été très étudiés afin de mieux comprendre la formation du rendement en matière sèche, il n'en est pas de même pour les racines. Or, celles-ci de par leurs multiples fonctions : ancrage et maintien de la plante, nutrition en eau et en éléments minéraux, secrétions hormonales, excrétion et formation de mucigel favorisant le développement des micro-organismes, mise en réserve sous forme de glucides notamment..., contribuent pourtant très fortement à l'élaboration du rendement final. L'amélioration de celui-ci, ainsi que la réduction des charges de culture (simplifications des façons culturales, réduction des apports d'engrais...) implique une meilleure connaissance du fonctionnement global de la plante, et plus particulièrement des mécanismes d'absorption hydrique et minérale.
Or, chez une plante, compte tenu de l'existence de corrélations internes et des variations des conditions pédo-climatiques, la position d'un organe a des incidences sur ses échanges avec les autres organes constitutifs du végétal et avec le milieu extérieur. L'étude de la structure constitue donc un préliminaire nécessaire à l'étude du fonctionnement.

L'enracinement d'une plante à un instant donné est fonction d'un double système d'interaction : entre la croissance et le développement du système racinaire, la croissance et le développement des parties aériennes d'une part, et le milieu pédo-climatique d'autre part.

Or, si l'existence de corrélations étroites entre la croissance et le développement des systèmes aériens et racinaires, dues à des régulations d'ordre nutritionnel et hormonal est connue de longue date (BROUwER \& DE WITT, 1969) l'absence de données précises à ce sujet ne permet souvent pas de dépasser le constat de relations d'allométrie entre masse des parties aériennes et masse des parties racinaires (TrOUGHTON, 1955). 
Pourtant, quelques travaux récents ont mis en évidence l'existence de liens très étroits entre le nombre de feuilles visibles et le nombre de racines primaires, chez le maïs (PICARD et al., 1985), chez le blé (KLEPPER et al., 1984), chez le riz (YAMAZAKI \& HARADA, 1982 ; PICARD, 1984). Il apparaît donc important de poursuivre ce type d'études de façon à pouvoir caractériser la structure de l'enracinement des plantes comme cela a été fait pour les parties aériennes (SHARMANN, 1942 ; FRANQUIN, 1974 ; GoUNOT et al., 1980).

A l'échelle de la parcelle existent des fluctuations importantes de densités racinaires dues à l'hétérogénéité des états structuraux du sol (TARDIEU, 1984) et à la compétition entre plantes au niveau d'un peuplement (étudiée en ce qui concerne les parties aériennes par GLENN \& DAYNARD, 1974 ; MASLE-MEYNARD \& SebillotTE, 1981 : Daynard \& Muldoon, 1983). Il importe donc de mieux caractériser l'évolution dans le temps et les variations dans l'espace de la structure du système racinaire (profondeur, distance maximale entre racines et point du profil). La compréhension des effets induits par les facteurs de milieu constitue une étape préliminaire indispensable à cette étude. La pauvreté de nos connaissances en ce domaine est attestée par le fait que l'ensemble des modèles de nutrition existants (TSCHESCHKE \& Gilley, 1979 ; Hillel \& TALPAZ, 1976 ; Wright \& KEENER, 1982) néglige ce problème. Ces auteurs considèrent que la colonisation racinaire est régulière et la maille élémentaire assez fine pour permettre la ponction, par le végétal, de l'ensemble des réserves du sol. Ceci est démenti, du moins en ce qui concerne l'eau, par les récents travaux de TARDiEu (1984).

En préambule à une étude de la dynamique racinaire du maïs au champ, ce travail a pour objet de dégager les paramètres descriptifs de la structure racinaire plus particulièrement sous la dépendance de la plante. Une seconde étude, complémentaire à celle-ci, permettra de décrire l'influence de quelques facteurs édaphiques, trophiques et climatiques. Les problèmes posés par l'intégration de ces données dans un modèle de structure cohérent seront également abordés au cours de cette seconde étape.

Cependant, les résultats obtenus sur le maïs sont très fragmentaires et concernent surtout le nombre de racines et l'évolution des matières sèches. Une étude de l'ensemble des paramètres descriptifs de l'enracinement nous oblige à faire référence à d'autres graminées dont la structure est comparable à celle du maïs. Dans le cas des plantes pérennes, nous ne considérerons que la phase d'implantation de la culture.

Nous ne nous attacherons pas à l'étude des conséquences des modifications d'équilibres hormonaux et de fonctionnement enzymatique difficiles à déceler au cours d'une étude de terrain. Il en est de même en ce qui concerne les processus de formation des ébauches, invisibles à l'œil nu.

Pour FRANQUIN (1974), la structure est définie par l'agencement des parties d'un système. C'est une composante abstraite de l'architecture d'une plante qui se manifeste dans le nombre d'organes et dans leurs relations spatiales.

Elle évolue constamment dans le temps et résulte, à un instant donné, de la cinétique de croissance et de développement. La valeur instantanée des paramètres descriptifs de la structure (nombre, longueur et orientation des racines de tous ordres) sera fonction de l'évolution antérieure de ceux relatifs à la dynamique racinaire (taux d'émission, de ramification et de nécrose, vitesse d'élongation). C'est ainsi que, par exemple, la longueur totale du système pourra être déduite des taux d'émission et des durées et vitesses d'élongation. La caractérisation de l'enracinement implique donc un suivi de sa dynamique.

\section{RÉGULATION DE LA CROISSANCE ET DU DÉVELOPPEMENT DE L'ENSEMBLE DU SYSTĖME RACINAIRE. INFLUENCE DES FACTEURS INTERNES À LA PLANTE}

Le système racinaire du maïs est composé d'une séminale préformée dans la graine (ONDERDONK \& KetCHESON, 1972 ; HiCKS \& PeTERSON, 1978) et de racines adventives néoformées, venant s'insérer à la base de chacun des 8 à 10 premiers entre-nœuds (SharmanN, 1942 ; KiesselbaCH, 1949 ; PiCARD $e t$ $a l ., 1985)$. Chacune des primaires, directement rattachée à la tige, va avec ses ramifications d'ordre II et III composer un réseau racinaire (PICARD, 1976). Sur un pied, on aura autant de réseaux que de racines primaires allongées et ramifiées, soit environ 30 à 40 (PICARD et al., 1985).

De fait, après l'émission, chaque racine va être soumise à 2 mécanismes de régulation dont la résultante va déterminer soit, son allongement, soit sa nécrose :

- une régulation d'ordre «physique »: l'apex (incluant la coiffe, le point végétatif et la zone subapicale où les cellules ne sont pas encore différenciées) subira une pression, déterminée par les caractéristiques physiques du substrat (structure, humidité, porosité) qui va influer sur l'orientation et la croissance de la racine (Gill \& Miller, 1956; BARLeY, 1962 ; BARLEY \& GREACEN, 1967);

- une régulation physiologique, intervient au niveau de l'ensemble du système racinaire et de chaque réseau qui se développe de façon autonome en fonction de son environnement pédo-climatique direct (HESS, 1969 ; WIGHTMAN \& ThIMANN, 1980 ; WiGHTMAN et al., 1980).

Cette régulation physiologique va, à son tour, être fonction ;

- du patrimoine génétique agissant notamment sur l'importance de l'équipement enzymatique donc sur la capacité de synthèse de métabolites ;

- des conditions de milieu qui déterminent la vitesse de mise en place de l'appareil végétatif, donc l'importance de celui-ci à un instant donné ;

- de l'état de croissance et de développement de la plante, dont dépend le niveau de métabolites, susceptible de permettre une croissance ultérieure. Celle-ci sera à son tour régulée par les composantes du milieu.

En outre, il existe de multiples interactions entre les différents facteurs agissant sur la structure du système racinaire. Il est donc malaisé de classer ces derniers de façon stricte, et une discussion croisée des résultats obtenus est souvent obligatoire. De plus, l'effet pro- 
pre à chacun d'entre eux est difficile à isoler et à quantifier et les études présentées ne dégagent souvent que les tendances générales des effets induits sur les paramètres de l'enracinement.

La dernière remarque concerne les techniques de culture et les méthodes de mesure utilisées pour l'obtention des résultats. En effet, la structure racinaire est susceptible d'être modifiée, à la fois par le support utilisé (solution nutritive, sable ou argile expansée, terre...), par le volume mis à la disposition des racines et par le fait que les plantes étudiées sont isolées ou regroupées (PETTERSON et al., 1984). De plus, l'extension des résultats obtenus au champ à d'autres situations est très difficile car l'on manque de méthodes permettant de décrire de façon objective et quantitative l'état structural du profil cultural. Enfin, les différentes méthodes d'études du système racinaire introduisent toutes des biais spécifiques sur les résultats obtenus (SHURMAN \& GOEDEWAAGEN, 1971; BÖHM, 1979 ; KOPKE, 1981). Une partie de la variabilité importante des données présentées peut donc être imputée à la diversité des techniques de culture et des méthodes de mesure.

\section{A. Le patrimoine génétique}

Les travaux de MANGELdoRF \& GOODSEll (1929), SIEMENS (1929), SPENCER (1940), ont montré que pour les variétés de maïs, le poids, le diamètre et l'orientation des primaires ainsi que leur degré de ramification sont variables. Il semblerait, en outre, que les hybrides aient plus de racines sur l'entre-nœud 1 (EI) que les lignées pures. En ce qui concerne les entre-nœuds supérieurs, les hybrides ont plus de racines primaires que leurs parents, mais si l'on considère un ensemble de variétés, la différence est faible et non significative, sauf sur E1. Sur cet entre-nœud, le type de grains semble également jouer un rôle important. En effet, les variétés dentées y initient de 3 à 6 racines, alors que chez les maïs doux, ce chiffre varie de 1 à 3 .

Les différences observées entre variétés sont expliquées, dans le cas du blé (PINTHus, 1969), par le fait que la période précédant l'initiation florale est plus longue chez les variétés tardives, qui développent un nombre d'organes aériens et racinaires plus élevé, que chez les variétés précoces, bien que le nombre de racines primaires émises par talle reste stable. Ceci entraîne une variabilité des densités racinaires en fonction de la variété (KOPKE et al., 1982).

Dans le cas du maïs, la précocité pourrait également expliquer une partie des différences observées, essentiellement en ce qui concerne le nombre total de racines primaires (DERIEUX, comm. pers.).

D’une façon générale, le poids du patrimoine génétique est d'autant plus important que la variabilité observée dans les conditions de culture les plus fréquemment rencontrées est faible. Pour une espèce donnée, il dépend à la fois de la sélection naturelle qui s'est opérée avant sa domestication et de la sélection humaine ultérieure en vue de l'obtention de variétés bien différenciées pour un caractère donné comme la précocité ou le type de grains (PERNES, 1983, étude portant sur le riz). Si les variations observées quant au nombre d'organes restent faibles au sein d'un groupe de population de même origine géographique, elles sont beaucoup plus importantes d'un groupe à l'autre.

\section{B. La mise en place simultanée des parties racinaires et aériennes}

Au cours du cycle cultural, des relations d'allométrie successives relient les phénomènes de croissance, notamment l'augmentation des poids secs des parties aériennes et racinaires (TEISSIER, 1948 ; TrOUGHTON, 1955 ; Troughton, 1960). Ceci peut être expliqué chez les graminées fourragères (BEHAEGHE, 1972 ; 1975 ; 1981) par l'existence d'états d'inductions internes à la plante liés à l'application de stress comme le pâturage ou la coupe et aux conditions de milieu telles que la température ou la richesse du sol en éléments nutritifs. Les réponses à ces inductions sont fonction de l'histoire du végétal et varient d'une situation à l'autre. D'après Gillet et al. (1984), les états d'induction détermineraient un potentiel de croissance (accroissement de la matière sèche) des parties aériennes. "La croissance racinaire pouvant également avoir son propre programme induit, mais la réalisation de ce dernier serait limitée par le carbone laissé libre par la croissance aérienne " (idée déjà évoquée par BROUWER, 1962). Dans ce cas, il y aurait stockage dans les racines de glucides pouvant être remobilisés rapidement si l'appareil photosynthétique venait à être détruit.

D'une manière générale, l'évaluation des paramètres descriptifs du développement d'un végétal (rythmes d'émission et nombre d'organes), se heurte à moins de difficultés méthodologiques et est obtenue avec un niveau d'exactitude plus grand que les estimations de croissance. Les relations reliant le développement des systèmes aériens et racinaires sont donc plus précises. Celles obtenues à ce jour concernent surtout l'évolution du rapport nombre de feuilles/nombre de racines primaires, et sont linéaires et stables pour une même variété (voir ci-dessous). Les variations induites par les phénomènes d'induction auraient une influence plus importante sur la croissance que sur le développement.

La mise en place conjointe des 2 systèmes, aérien et racinaire, peut être appréhendée par le suivi de paramètres caractérisant le développement tels que :

\section{Les rythmes d'émission racinaire}

Ceux-ci sont très fortement corrélés au phyllochrone défini par GoUNOT et al. (1980), chez le blé (KLEPPER et al., 1984), le dactyle (El KHODRE, 1983) et le maïs (PICARD et al., 1985). Chez cette plante les racines de l'entre-nœud $X$ commencent à être émises quand la feuille du phytomère (défini par SHARMANN, 1942) $2 X$ émerge du cornet.

Chez le riz, on observe un décalage de 3 entrenœuds entre l'apparition des feuilles et celle des racines primaires. Cet écart passe respectivement à 4 et 5 entre-nœuds pour les secondaires et les tertiaires (YAMAZAKI \& HARADA, 1982). A la fin de l'émission foliaire, l'apparition des primaires à un niveau donné correspond à un stade de développement précis : l'initiation de la panicule pour le niveau 9, et sa différenciation pour le niveau 10 (KAWATA \& SoEJIMA, 1974). Les travaux de PICARD \& JACQUOT (1976) montrent 
cependant que ce schéma n'est pas généralisable aux variétés "africaines », qui n'ont pas la même origine que les variétés « japonaises», même si la relation entre rythmes d'émissions racinaire et foliaire est stable pour une variété donnée.

\section{Les nombres d'organes émis}

L'équation suivante montre la relation existant chez les variétés de riz cultivées au Japon entre le nombre de racines nodales primaires (Y) et le nombre d'épis (X) (YAMAZAKI et al., 1980) :

$$
\mathrm{Y}=28,3 \mathrm{X}+107,0 \quad \mathrm{r}=0,819 .
$$

Cette relation doit cependant présenter une certaine variabilité pour l'ensemble des variétés cultivées (PICARD \& JACQUOT, 1976).

D'autre part, MANGELdORF \& GoOdSElL (1929), NASS \& ZUBER (1971) ont constaté qu'au sein d'un peuplement de maïs, les plantes dont la vigueur germinative est faible et qui restent chétives tout au long du cycle ont plus de racines sur le mésocotyle que les pieds à développement normal. Cependant, on ne sait pas si la présence de ces primaires sur le mésocotyle est imputable à un accident de croissance ou à une anomalie de la semence qui va freiner le développement de la plante.

L'étude des paramètres suivants montre qu'il y a synchronisme de croissance entre les systèmes aériens et racinaires.

\section{La taille des organes}

Chez le riz, le diamètre des racines primaires est relié à celui de leur entre-nœud d'insertion (MORITA et al., $1983 a$ et $b$ ). La relation obtenue est stable pour les variétés japonaises. De tels travaux n'ont pas encore été entrepris pour le maïs.

Si l'on considère le système dans son ensemble, l'idée classiquement admise que le volume colonisé par les parties aériennes est proportionnel à celui occupé par les racines (WEAVER \& CLEMENTS, 1923 dans Evans, 1973 ; Troughton, 1957 dans Evans, 1973) est à nuancer, compte tenu des variations importantes de la structure du système racinaire induites par la structure et l'humidité du sol.

Pour le maïs (FoTH, 1962) on distingue cependant 5 stades successifs au cours du cycle. Ces stades sont déterminés à partir des états de développement des systèmes aériens et racinaires : le premier se termine quand apparaissent les racines adventives; la seconde phase est définie comme étant la période de croissance végétative pour les parties aériennes. Durant cette période, les horizons superficiels vont être colonisés de façon préférentielle, les horizons profonds le seront durant la phase suivante, marquée par l'émission des organes reproducteurs et la floraison. La croissance racinaire va se terminer pendant la maturation du grain qui constituera la $4^{\mathrm{e}}$ étape. Puis en fin de cycle, il $\mathrm{y}$ a début de nécrose racinaire et diminution du volume occupé par les racines.

CHOLICK et al. (1977) ont montré que chez le blé cultivé, dans une situation pédo-climatique donnée, l'importance du système racinaire (biomasse et profondeur d'enracinement) était proportionnelle à la hauteur de la plante. Les différences observées sont faibles au niveau d'un peuplement homogène mais importantes entre variétés.

Chez le riz, enfin, le rendement serait très fortement corrélé à la densité racinaire dans les 30 premiers $\mathrm{cm}$ de sol (KAWATA et al., 1978). Ceci peut s'expliquer par le fait qu'un niveau élevé de fertilisation azotée favorise et la ramification racinaire et la croissance des parties aériennes.

L'ensemble de ces travaux permet de dégager des synchronismes de croissance et de développement entre les systèmes aériens et racinaires. Ces tendances prédéterminées vont être plus ou moins marquées en fonction des facteurs constitutifs du complexe climatsol-plante, comme cela a été montré par GILLET et al. (1984).

\section{Les vitesses d'élongation}

En conditions artificielles avec une alternance journuit de 2 températures constantes, l'accroissement en matière sèche des parties aériennes est exponentielle et la croissance racinaire linéaire (LAMBERS et al., 1982). En conditions naturelles, les relations ne sont pas aussi nettes mais, pour beaucoup de plantes cultivées, la croissance est corrélée positivement à l'éclairement, à l'indice de couverture foliaire et à la température, comme le montre la revue faite par LAMBERS (1983).

\section{Les poids secs aériens ( $P S A$ ) et racinaires (PSR)}

La relation entre les 2 grandeurs est linéaire en début de croissance (BrouwER et al., 1981) puis exponentielle jusqu'après floraison (BROUWER, 1967, revue générale). Le rapport PSA/PSR baisse ensuite en fin de cycle. On a, en fait, plusieurs relations d'allométrie successives, correspondant chacune à une phase de développement précise de la plante. Les changements se font à des périodes critiques, comme l'initiation florale pour l'ensemble des graminées. La croissance et le développement des primordias floraux nécessitant une quantité importante de carbohydrates, ce stade correspond à une augmentation importante du rapport $\mathrm{C} / \mathrm{N}$ de l'ensemble de la plante. De plus, l'accroissement en matière sèche est beaucoup plus important pour l'appareil aérien que pour le système racinaire, et le rapport PSA/PSK est tortement modifié (Troughton, 1955 ; Troughton, 1960).

La valeur de ce rapport est en outre déterminée par l'espèce et les conditions du milieu : une carence minérale réduit ce rapport (JAGER, 1982) alors qu'un éclairement faible produit un effet inverse (BROUWER, 1963 dans PICARD, 1976 ; WILKINS \& WaIN, 1975).

De plus, en culture hydroponique, toute ablation modifie le rapport PSA/PSR et perturbe les vitesses d'élongation de façon à compenser le stress induit. Une mutilation foliaire, en réduisant la photosynthèse, donc la fourniture de glucides aux racines, diminue leur croissance. Tant que les 2 systèmes resteront en compétition pour l'énergie, donc que le rapport initial PSA/PSR ne sera pas rétabli, le développement du système racinaire sera retardé. L'excision racinaire, en réduisant la fourniture d'eau et de nutriments aura un effet inverse, et fera baisser la croissance des parties aériennes, ce qui peut avoir un effet pénalisant sur le rendement (BOATWRIGHT \& FERGUSSON, 1967 ; BROUWER, 1967). 
Les conditions de milieu jouent un rôle déterminant comme pour l'ensemble des paramètres descriptifs de la croissance. Par contre, les relations reliant les phénomènes de développement des systèmes aériens et racinaires sont beaucoup plus stables, donc moins dépendantes des conditions pédo-climatiques.

\section{Régulation interne au système racinaire}

Chaque réseau se développe de façon indépendante en fonction :

\section{Du diamètre de la racine primaire}

Des travaux récents portant sur le riz (KAWATA et $a l ., 1980$ ) et sur le maïs (YAMAZAKI \& KAERIYAMA, 1982) ont permis de mettre en évidence la présence de 3 catégories de primaires. On distingue, dans l'ordre, des racines à diamètre faible et constant issues des premiers entre-nœuds, des racines plus grosses dont le diamètre varie peu, insérées aux entre-nœuds intermédiaires et enfin, des primaires dont le diamètre, important à la base, décroît ensuite rapidement et qui sont portées par les derniers niveaux. Le nombre, par unité de longueur, de secondaires issues de ces mêmes racines est d'autant plus important que l'on passe d'une catégorie à la suivante. Leur diamètre est de plus corrélé à celui des primaires porteuses.

Les travaux sur le maïs de PICARD et al. (1985), permettent d'apporter une précision supplémentaire. Ces auteurs trouvent en effet une relation linéaire entre le diamètre des racines primaires pris à la base et le numéro de l'entre-nœud (déterminé selon la terminologie d'ONDERDONK \& KETCHESON, 1972, et de GIRARDIN $e t a l .$, 1986) sur lequel elle est insérée.

Sur le riz, on a, en outre, mis en évidence une relation entre le diamètre racinaire, l'angle de croissance et le nombre de vaisseaux du bois (YAMAZAKI et al., 1981 ; MORITA et al., 1983a et $b$ ). Ce travail n'a pas encore été fait sur le maïs.

\section{Du développement antérieur du système}

PAVlChENKo (1937) constate que la longueur racinaire totale du maïs est corrélée positivement à la longueur du système séminal et ce, sur des sols de texture et niveaux de densité apparente différents. Chez cette même plante, le poids racinaire est d'autant plus faible que le nombre de racines portées par le nœud scutellaire est élevé (NASS \& ZUBER, 1971).

Pour la plupart des céréales, une ablation des racines primaires ne modifie pas le nombre de secondaires initiées sur les racines primaires restantes, mais va provoquer une augmentation du nombre de nodales sur les entre-nœuds supérieurs, où l'émission n'a pas encore débuté au moment de l'excision (HACKETT, 1971 ; CROSSET et al., 1975).

\section{Du développement simultané des autres parties $d u$ système}

Tout se passe comme si chaque réseau se comportait en fonction de son environnement pédoclimatique direct, mais de façon à assurer un développement optimal de l'ensemble du système. C'est ainsi qu'une privation nutritionnelle localisée aux $3 / 4$ de l'appareil racinaire ne modifie pas le poids sec racinaire total, mais induit une croissance et une ramification plus rapides des racines en contact avec les nutriments manquants, alors que le reste du système ne s'allonge pas. La vitesse d'absorption des métabolites impliqués $\left(\mathrm{K}^{+} ; \mathrm{Ca}^{++} ; \mathrm{PO}^{3}{ }_{4}^{-}\right)$est accélérée (JENNY, 1961 ; BALDIN \& NYE, 1974 ; JAGER, 1984).

\section{DISCUSSION - CONCLUSION}

A la lumière de cette étude, il apparaît que l'état de nos connaissances de la structure racinaire et plus particulièrement des relations existants entre la cinétique de mise en place des systèmes racinaires et aériens demeure très fragmentaire.

Ceci est imputable à 3 causes :

- Les méthodes classiques d'investigation ne permettent pas de suivre la cinétique de croissance et de ramification en profondeur. De plus elles sont destructives et sujettes à des biais spécifiques et les résultats obtenus ne sont pas toujours concordants.

- D'autre part, peu d'auteurs ont essayé de définir la structure du système racinaire et les paramètres qui permettent de le décrire précisément. Les études entreprises ont rarement eu pour objet le système racinaire dans sa totalité. Même les modèles cinétiques ne s'intéressent qu'à 1 ou 2 de ses composantes comme la biomasse ou le nombre d'organes.

- Enfin, l'étude de l'influence des conditions pédoclimatiques et la recherche de correlations entre la croissance et le développement du système racinaire ont souvent été dissociées. Le rôle des interactions entre les facteurs constitutifs du complexe climat-solplante, prépondérantes en conditions de grande culture, est encore peu connu.

Les conséquences de cet état de fait sur notre travail ont été multiples.

Les références sur le maïs étant peu nombreuses, nous avons élargi notre champ d'investigation à d'autres graminées qui, comme le maïs, sont composées d'une série d'unités élémentaires superposées : chacune d'entre elles comprenant un nœud et un entrenœud, une gaine et un limbe, un bourgeon axillaire susceptible d'évoluer en talle, et, à la base de chacun des entre-nœuds une rangée de bourgeons pouvant donner naissance à des racines primaires. On peut donc penser, a priori, que des analogies existent également au niveau du système racinaire. Dans ce cas, il est possible d'établir des hypothèses sur la structure du maìs à partir des résultats obtenus sur d'autres graminées. Ces hypothèses, devant bien sûr être vérifiées expérimentalement au cours d'une étape ultérieure.

Les travaux cités concernent principalement la biomasse et la longueur des racines, plus rarement le nombre de racines primaires. Seul un petit nombre d'auteurs (HAKETT \& ROSE, 1971 ; HAKETT \& ROSE, $1972 a$; LUNGLEY, 1973 ; WANG, 1983) ont défini les paramètres nécessaires à la description de l'ensemble du système racinaire. A l'exception du modèle de WANG (1983), qui s'adresse à de jeunes plantules de maïs dont la longueur n'excède pas $10 \mathrm{~cm}$, ces travaux sont restés purement théoriques. L'état actuel de nos connaissances ne nous permet donc pas de modéliser 
la cinétique de croissance et le développement du système racinaire en fonction de celle du système aérien, et encore moins d'améliorer la précision des modèles de nutrition existants. Tout au plus pouvonsnous, en ce qui concerne l'allocation des hydrates de carbone, l'absorption d'eau et d'éléments nutritifs, raisonner en termes de réseaux racinaires définis, par PICARD (1976) ou de rang de l'entre-nœud d'insertion des racines primaires. Nos lacunes concernant les racines d'ordre supérieur ou égal à 2 posent également des problèmes pour l'évaluation de la masse de ces ordres racinaires. D'autre part, ce sont ces organes dont le diamètre est faible qui colonisent les pores les plus fins et pénètrent à l'intérieur des agrégats. Leur rôle pour l'absorption d'éléments faiblement mobiles comme le phosphore et le potassium semble très important. De plus, leur prise en compte est déterminante dans l'évaluation des distances entre racines et points du profil, variable classiquement utilisée dans les modèles d'absorption.

Rappelons enfin que les résultats présentés ici ont été obtenus en grande partie en conditions contrôlées susceptibles de modifier la structure racinaire, et qu'ils peuvent difficilement être extrapolés en champ, niveau d'étude que nous nous sommes fixés et qui intéresse plus particulièrement l'agriculteur. Dans ce cas également, des expérimentations complémentaires sont nécessaires.

Compte tenu des interactions entre les différents facteurs tant internes, qu'externes à la plante, et de leur influence simultanée sur la mise en place du système racinaire, il est impossible de classer les paramètres descriptifs de la structure de façon stricte en 2 catégories : ceux sous la dépendance de la plante, et ceux sous la dépendance du milieu. Cependant il semble que les paramètres caractéristiques du développement, comme le nombre d'organes, soient beaucoup plus liés au stade de croissance et au développement du végétal qu'aux conditions pédo-climatiques. Les vitesses de croissance et les tailles des organes sont quant à elles, sous la dépendance prépondérante de l'environnement pédo-climatique.

Pour pouvoir être utilisé avec profit dans l'étude de la dynamique racinaire au champ, ce travail doit obligatoirement être complété par une étude de l'influence des facteurs constitutifs du complexe climat-sol sur la structure racinaire (JORDAN, 1987).

Reçu le 11 juin 1986. Accepté le 10 mars 1987.

\section{RÉFÉRENCES BIBLIOGRAPHIQUES}

Baldwin J.-P., Nye P. H., 1974. A model to calculate the uptake by a developing root system or root hair system of solutes with concentration variable diftuston coettcients. Plant soul, 40, 703-706.

Barley K. P., 1962. The effect of mechanical stress on the growth of roots. J. exp. Bot., 13 (37), 97-110.

Barley K. P., Greacen E. I., 1967. Mechanical resistance as a soil factor influencing the growth of roots and underground shoots. Adv. Agron., 19, 1-43.

Behaeghe T. J., 1972. De bewortelingsdichtheid onder grasland en haar gevolgen vor de watervoorsiening. Agricultura, Louvain, 20 (3), 149-175.

Behaeghe T. J., 1975. Eco-fysiologische grondslagen van de grasgoei. Agricultura, Louvain, 23 (4), 375-392.

Behaeghe T. J., 1981. Exploitation des prairies. 4. La variation dans la croissance de l'herbe ; causes et conséquences pour l'exploitation des prairies. Rev. agric., 34 (3), 511-515.

Boatwright G. O., Ferguson H., 1967. Influence of primary and/or adventitious root systems on wheat production and nutrient uptake. Agron. J., 359, 299-302.

Böhm W., 1979. Methods of studying root systems. Ecological studies 33, Springer verlag $188 \mathrm{p}$.

Brouwer R., 1962. Nutritive influences on the distribution of dry matter in the plant. Neth. J. agric. Sci., 10 (5), 399-408.

Brouwer R., 1967. Bezicluungen zwischen Spross und Wurzelwachstum. Angew. Bot., 41, 244-254.

Brouwer R., de Witt C. T., 1969. A simulation model of plant growth with special attention to root growth and its consequences. In W. J. Whittington Root Growth, 224-244, Butterworths, London.

Brouwer R., Gasparikowa O., Koleh J., Loughman B. C., 1981. Structure and function of plant roots: Proceedings of the 2nd Symposium held in Bratislava, Czechoslovakia, Sept. 1-5, 1980. Martinus Nijhoff, London.

Cholick F. A., Welsh J. R., Vernon-Cole C., 1977. Rooting patterns of semidwarf and tall winter wheat cultivars under dryland field conditions. Crop Sci., 17, 637-639.

Crosset R. N., Campbell D. J., Stewart H. E., 1975. Compensatory growth in cereal root systems. Plant Soil, 42, 673-683.
Daynard T. B., Muldoon J. F., 1983. Plant-to-plant variability of maize plants grown at different densities. Can. J. Plant Sci., 68, 4559.

EI Khodre A., 1983. Interaction chez, le dactyle (Dactylis glomerata L.) de la croissance et du développement végétatif avec l'alimentation en eau et en azote. Application aux bilans hydrique et énergétique à la productivité d'une culture. Thèse de Doctorat d'Etat, ULP Botanique, Strasbourg, $447 \mathrm{p}$.

Evans P. S., 1973. Plant root distribution and soil-water extraction. Proc. Soil Plant Wat. Symp., DSIR Inform., Ser., 96, 5-8.

Foth H. D., 1962. Root and top growth of corn. Agron. J., 54 (1), 49-52.

Franquin P., 1974. Un modèle théorique du développement de la structure de la plante. Physiol. veg., 12 (3), 459-465.

Gill W. K., Miller R. D., 1956. A method for study of the influence of mechanical impedance and aeration on the growth of seedling roots. Soil Sci. Soc. Am. Proc., 20, 154-157.

Gillet M., Lemaire G., Gosse G., 1984. Essai d'ćlaboration d'un schéma global de la croissance des graminées fourragères. Agronomie, 4 (1), 75-82.

Girardin Ph., Jordan M.-O., Picard D., Trendel R., 1986. Harmonisation des notations concernant la description morphologique d'un pied de maïs. Agronomie, 6 (1), 873-875.

Glenn F. B., Daynard T. B., 1974. Effects of genotype, planting pattern, and plant density on plant-to-plant variability and grain yield of corn. Can. J. Plant Sci., 54, 323-330.

Gounot M., Atry M., N'Kandza J., Yu O., 1980. Photosynthèse nette et rythme d'apparition des feuilles chez le dactyle (Dactylis glomerata L.). C.R. Acad. Sci. Paris, 290 D, 1257-1260.

Hackett C., 1971. Relations between the dimensions of the barley root system : effects of mutilating the root axes. Aust. J. biol. Sci., 24, 1057-1064.

Hackett C., Rose D. A., 1972a. A model of the extension and branching of a seminal root of barley and its use in studying relations between root dimensions. I. The model. Aust. J. biol. Sci., 25, 669679.

Hackett C., Rose D. A., 1972b. A model of the extension and branching of a seminal root of barley and its use in studying relations 
between root dimensions. II. Resultats and inferences from manipu lation of the model. Aust. J. biol. Sci., 25, 681-690.

Hess C. E., 1969. Internal and external factors regulating root initiation. In W. J. Whittington Root Growth, 42-54, Butterworths, London.

Hicks D. R., Peterson R. H., 1978. Lower stalk development and staging of corn. Agron. J., 70, 485-488.

Hillel D., Talpaz H., 1976. Simulation of root growth and its effect of the pattern of soil water uptake by a non-uniform rool system. Soil Sci., 121, 307-312.

Jager (de) A., 1982. Effects of a localized supply of $\mathrm{H}_{2} \mathrm{PO}_{4}, \mathrm{NO}_{3}$, $\mathrm{SO}_{4}, \mathrm{Ca}$ and $\mathrm{K}$ on the production and distribution of dry matter in young maize plants. Neth. J. agric. Sci., 30, 193-203.

Jager (de) A., 1984. Effects of a localized supply of $\mathrm{H}_{2} \mathrm{PO}_{4}, \mathrm{NO}_{3}$, $\mathrm{Ca}$ and $\mathrm{K}$ on the concentration of that nutrient in the plant and the rate of uptake by roots in young maize plants in solution culture. Neth. J. agric. Sci., 32 (1), 43-56.

Jenny, 1961. Plant soil interactions. In Zarrow et al. Plant living systems, 665-694, Basic Bodetime, New York.

Jordan M.-O., 1987. Mise en place du système racinaire du maïs II. Importance de quelques paramètres relatifs aux conditions de milieu. Agronomie, 7 (7).

Kawata S., Soejima M., 1974. On superficial root formation in rice plants. Proc. Crop. Sci. Soc., Jpn, 43 (3), 354-374.

Kawata S., Soejima M., Yamazaki L., 1978. The superficial root formation and yield of hulled rice. Jpn J. Crop Sci, 47 (4), 617628 .

Kawata S., Sasaki O., Yamazaki K., 1980. On the lateral root formation in relation to the diameter of crown roots in rice plants. Jpn J. Crop Sci., 49 (1), 103-111.

Kiesselbach T. A., 1949. The structure and reproduction of corn. University of Nebraska Press., Reed. 1980, $96 \mathrm{p}$.

Klepper B., Belford R. K., Rickmann R. W., 1984. Root and shoot development in winter wheat. Agron. J., 76 (1), 117-123.

Kopke U., 1981. A comparison of methods for measuring root growth of field crops. Z. Acker Pflanzenbau, 150, 39-49.

Kopke U., Bohm W., Jachmann Th., 1982. Rooting patterns of three winter wheat cultivars in a field and greenhouse experiment. Z. Acker Pflanzenbau, 151, 42-48.

Lambers H., 1983. " The functional equilibrium ", nibbling on the edges of a paradigm. Neth. J. agric. Sci., 31 (4), 305-311.

Lambers H., Simpson R. J., Beilharz W. C., Dalling M. J., 1982. Growth and translocation of $\mathrm{C}$ and $\mathrm{N}$ in wheat (Triticum aestivum) grown with a split root system. Physiol. Plant., 56, 421-425.

Lungley D. R., 1973. The growth of root systems. A numerical computer simulation model. Plant Soil, 38, 145-159.

Mangeldorf P. C., Goodsell S. F., 1929. The relation of seminal roots in corn to yield and various seed, ear and plant caracters. $J$. am. Soc. Agron., 21, 52-68.

Masle-Meynard J., Sebillotte M., 1981. 1. Notion de structure de peuplement. Agronomie, 1 (3), 207-216.

Morita S., Yamazaki K., Kawata S., 1983a. Relationships between the growth direction of primary roots and their anatomical characters in rice plants. Jpn J. Crop Sci., 52 (4), 551-554.

Morita S., Yamazaki K., Kawata S., 1983b. Relationships between the growth direction of primary roots and their conductive capacities in rice plants. Jpn J. Crop Sci., 52 (4), 562-566.

Nass H. G., Zuber M. S., 1971. Correlation of corn (Zea mays L.) roots early in development to mature root development. Crop Sci., $11,5,655-658$.

Onderdonk J. J., Ketcheson J. W., 1972. A standardization of terminology for the morphological description of corn seedlings. Can J. Plant Sci., 52, 1003-1006.

Pavlichenko T. K., 1937. Quantitative study of the entire root systems of weed and crop plants under field conditions. Ecology, 18 (1), 62-79.

Pernes J., 1983. La génétique de la domestication des céréales. Recherche, 146 (14), 910-919.

Petterson C. M., Klepper B., Pumphrey F. V., Rickmann R. W. 1984. Restricted rooting decreases tillering and growth of winter wheat. Agron. J., 76 (5), 861-863.
Picard D., 1976. Dynamique racinaire de Panicum maximum. Jacq. et apport au sol de matière organique. Thèse Dout.-Ing. es-Sci., Clermont-Ferrand, $275 \mathrm{p}$.

Picard D., 1984. Etude racinaire et résistance à la sécheresse. Comm. Coll. «Résistance à la sécheresse en milieu intertropical. Quelles recherches pour le moyen terme? Dakar, 24-27.09.1984, 131-143.

Picard D., Jacquot M., 1976. Rythmes d'émission comparés des racines nodales de trois variétés de riz. Agro. trop., 31, 159-169.

Picard D., Jordan M.-O., Trendel R., 1985. Rythme d'apparition des racines primaires du maîs. 1. Etude pour une variété en un lieu donné. Agronomie, 5 (8), 667-676.

Pinthus M. J., 1969. Spread of root system as indicator for evaluating lodging resistance of wheat. Crop Sci., 7, 107-110.

Ricard J., 1960. La croissance des végétaux. «Que sais-je? » PUF $\mathrm{n}^{\circ} 898$, Paris, $126 \mathrm{p}$.

Sharmann B. C., 1942. Developmental anatomy of the shoot of Zea mays. Ann. Bot., 6 (22), 245-282.

Shurman J. J., Goedewaagen M. A. J., 1971. Methods for the examination of root systems and roots. Center for agricultural publishing and documentation, Wageningen, $86 \mathrm{p}$.

Siemens H. J., 1929. The development of secondary roots in corn seedlings. Sci. Agric., 9, 747-759.

Spencer J. T., 1940. A comparative study of the seasonal root development of some inbred lines and hybrids of maize. J. agric. Res., 61 (7), 521-538.

Tardieu F., 1984. Etude au champ de l'enracinement du mais. Influence de l'état structural sur la répartition des racines. Conséquences sur l'alimentation hydrique. Thèse Docteur-Ingénieur, INA-PG, $232 \mathrm{p}$.

Teissier G., 1948. La relation d'allométrie, sa signification statistique et biologique. Biometrics, 4, 14-53.

Troughton A., 1955. The application of the allometric formula to the study of the relationship between the root and shoots of grass plants. Agric. Prog., 30, 59-65.

Troughton A., 1960. Further studies on the relationship between shoot and root systems of grasses. J. br. Grassl. Soc., 15 (1), 41-47.

Tscheschke P. D., Gilley J. R., 1979. Status and verification of Nebraska's corn growth model : CORNGRO. Trans. ASAE, 22 (6), 1329-1337.

Wang J., 1983. Growth simulation of the corn radicle root for various soil physical condition. Ph. D. Pennsylvania State University, 1949, $193 \mathrm{p}$.

Wightman F., Thimann K. W., 1980. Hormonal factors controlling the initiation and development of lateral roots. I. Sources of primordia-inducing substances in the primary root of pea seedlings. Physiol. Plant., 49, 13-20.

Wightman F., Schneider E. A., Thimann K. W., 1980. Hormonal factors controlling the initiation and development of lateral roots. II. Effects of exogenous growth factors on lateral root formation in pea root. Physiol. Plant., 49, 304-314.

Wilkins H., Wain R. L., 1975. The role of the root cap in the response of the primary roots of Zea mays $\mathrm{L}$. seedlings to white light and to gravity. Planta, 12 (3), 217-222.

Wright A. D., Keener M. E., 1982. A test of a maize growth and development model, CORNF Agric. Syst., 9, 181-197.

Yamazaki K., Katanom., Kawata S., 1980. The relationship between the number of ears and the number of crown roots on a hill of rice plants. Jpn J. Crop Sci., 49 (2), 317-322.

Yamazaki K., Morita S., Kawata S., 1981. Correlations between the growth angles of crown roots and their diameters in rice plants. Jpn J. Crop Sci., 50 (4), 452-456.

Yamazaki K., Harada J., 1982. The root system formation and its possible bearing on grain yield in rice plants. Jpn J. Crop Sci., 15 (3), $153-160$

Yamazaki K., Kaeriyama N., 1982. The morphological characters and the growing directions of primary roots of corn plants. Jpn $J$. Crop Sci., 51 (4), 584-590. 